\title{
Protective effect of dexmedetomidine against myocardial ischemia-reperfusion injury in rabbits ${ }^{1}$
}

\author{
Jianjun Ren', Changfa Li", Yan Liu'"', Haitao Liu'v , Zhenming Dong ${ }^{\vee}$
}

'PhD, Department of Anesthesia, the Second Hospital of Hebei Medical University, Hebei, China. Designed the protocol, technical procedures, acquisition of data, statistical analysis, manuscript writing.

"Bachelor, Department of Anesthesia, Zaozhuang Maternity and Child Care Centers, Shandong, China. Technical procedures.

"'Master, Department of Anesthesia, the Third Hospital of Hebei Medical University, Hebei, China. Technical procedures. IVPhD, Department of Anesthesia, the Second Hospital of Hebei Medical University, Hebei, China. Technical procedures. ${ }^{\vee} \mathrm{PhD}$, Department of Anesthesia, the Second Hospital of Hebei Medical University, Hebei, China. Designed the protocol.

\begin{abstract}
Purpose: To investigate the influence of dexmedetomidine on myocardial ischemiareperfusion injury (IRI) in rabbits.

Methods: Twenty-four New Zealand white rabbits were randomly divided into two equal-sized groups: IRI group (group IR) and dexmedetomidine group (group D). Systolic blood pressure (SBP), diastolic blood pressure (DBP), heart rate (HR), left ventricular systolic pressure (LVSP), left ventricular end-diastolic pressure (LVEDP), left ventricular diastolic pressure (LVDP), +dp/ dtmax,-dp/dtmax, and t-dp/dtmax were recorded and calculated the following time points: before (TO) and after (T1) dexmedetomidine infusion, after 30-min ischemia (T2), and after 120-min reperfusion (T3). The levels of plasma endothelin 1 (ET-1), thromboxane A2 (TXA2), and platelet activating factor (PAF); area of myocardial infarction (MI); and no-reflow area were evaluated.

Results: SBP, DBP, LVSP, LVEDP, LVDP, and $+d p / d t m a x$ at T3 were higher in group $D$ than in group IR $(P<0.05)$. The average no-reflow area in group IR was significantly smaller than that in group $D(14 \pm 3 \%$ vs. $38 \pm 5 \%, P=0.0116)$. The $E T-1, T X A 2$, and PAF levels at T2 and T3 were higher than those at $\mathrm{TO}$ in both groups $(\mathrm{P}<0.05)$.

Conclusion: Dexmedetomidine could reduce the magnitude of ischemic myocardial noreflow area and protect the myocardium with ischemia-reperfusion injury.

Key words: Dexmedetomidine. Heart Function Tests. Myocardial Reperfusion Injury. Rabbits.
\end{abstract}




\section{Introduction}

Myocardial ischemia-reperfusion injury (IRI) is a common pathological phenomenon occurring with myocardial infarction (MI) treatment, resulting in ischemia of cardiomyocytes and myocardial microvessel injury ${ }^{1,2}$, the mechanism of which involves events such as damage to vascular endothelial cells, activation of inflammatory factors and alexins, and thrombosis, among others ${ }^{3,4}$. After blood flow recovery in the myocardium with acute ischemia, part of the ischemic myocardial tissue receives incomplete or no perfusion, termed the "no-reflow phenomenon". Previous studies have indicated that vascular endothelial injury ${ }^{4}$, blood platelets, and inflammatory mediators were the main factors involved in this phenomenon ${ }^{6}$. The no-reflow phenomenon has been shown to occur in both animal experiments and cardiac revascularization surgery ${ }^{7,8}$, and its reduction will significantly reduce the $\mathrm{Ml}$ area and myocardiac remodeling and improve patients' prognosis ${ }^{9}$.

Various clinical strategies have been adopted to reduce myocardial noreflow, among which drug intervention is important $^{10,11}$. Dexmedetomidine is a highly selective $\alpha 2$ receptor agonist that can protect the myocardium, as proven in an animal experiment and a clinical study ${ }^{12,13}$. Owing to the wide distribution of the $\alpha 2$ receptor in the cardiovascular, central, and peripheral nervous systems, as well as in blood platelets, it could exert multiple biological effects within the human body ${ }^{14}$. Previous studies could not accurately evaluate the direct effect of dexmedetomidine on the myocardia with IRI with the use of in vitro myocardial ischemia reperfusion models or with in vivo models when only indirect parameters were monitored. Therefore, in this study, an in vivo model was applied to assess how dexmedetomidine influences the myocardia with IRI and to further investigate the possible mechanism.

\section{Methods}

\section{Preparation of the myocardial IRI model}

This study was carried out in strict accordance with the recommendations in the Guide for the Care and Use of Laboratory Animals of the National Institutes of Health. The animal use protocol has been reviewed and approved by the Institutional Animal Care and Use Committee (IACUC) of Hebei Medical University.

A total of 24 clean male New Zealand white rabbits weighing $2.3-2.6 \mathrm{~kg}$ were provided by the Laboratory Animal Center, Academy of Agricultural Sciences, Shandong Province. After the injection of $30 \mathrm{mg} / \mathrm{kg}$ pentobarbital sodium into an ear vein of the rabbit, its trachea was incised and a cannula was intubated into the opening, maintaining spontaneous breathing with pure oxygen. Electrocardiograph monitoring was performed for each rabbit. Systolic arterial pressure (SAP), diastolic arterial pressure (DAP), and heart rate (HR) were monitored through femoral artery cannulation, whereas central venous pressure (CVP) was monitored through right jugular vein cannulation. A catheter for atrial pressure measurement was inserted into the left auricle and tied with ligature. The left common carotid artery was separated and a catheter for left atrial pressure (LAP) measurement was inserted into it until $4-5 \mathrm{~cm}$. Ventricular wave could be observed when the catheter reached the left ventricle. All catheters were filled with heparin sodium $(20 \mathrm{IU} / \mathrm{mL})$ for anticoagulation. All parameters demonstrating left atrial and ventricular functions were measured using a YP100 pressure transducer connected with a RM6240 multichannel physiological 
signal detector (Chengdu Instrument Factory; parameter setting: data sampling frequency, $800 \mathrm{~Hz}$; scanning speed, $250 \mathrm{~ms} /$ div; calibration sensitivity, $90 \mathrm{mmHg}$; time constant, direct current; filter frequency, 30 $\mathrm{Hz}$ ). The thoracotomy was started by incising close to the left edge of the sternum and then clipping two to three ribs. After opening the chest cavity, the pericardium was gently lifted and incised to expose the heart, and the LAP was measured using the catheter through the left auricle. The left ventricular branch (LVB) of the left circumflex branch of the left coronary artery (LCA) was exposed by lifting the left auricle gently with hemostatic forceps to turn the heart to the right. At the midpoint of this artery, a 5-0 suture was threaded through the tissue surrounding the artery, to encircle the artery together with a 4-5-cm-long thin catheter with a small-eyed needle for further study, and a pouch suture was performed in the left auricle. Finally, the catheter was inserted to monitor the LAP. After all the procedures were done, the heart was left to stabilize for $30 \mathrm{~min}$.

\section{Grouping}

After the threading operation through the LVB of the LCA, the animals were randomly divided into the IRI group (group IR) and the dexmedetomidine group (group D). In group $I R$, the rabbits were intravenously infused with normal saline into the marginal ear vein, with the same speed as those in group $D$ for $20 \mathrm{~min}$. After infusion, myocardial ischemia was generated by sliding the catheter along the suturing line, tightening it, and fixing with hemostatic forceps. The color of the ischemic area turned to cyan and then purple, and S-T elevation ( $>0.5 \mathrm{mV}$ ) was observed, indicating successful ischemia. After ischemia was maintained for $30 \mathrm{~min}$, the ligature was loosened to recover blood perfusion. An S-T depression $(>1 / 2)$ indicated that reperfusion was achieved, which was maintained for 120 min. In group D, $200 \mu$ g dexmedetomidine was dissolved in $50 \mathrm{~mL}$ normal saline and infused. The infusion time was $20 \mathrm{~min}$, with a final dose of $2.75 \mu \mathrm{g} / \mathrm{kg}$ for each rabbit, followed by $30-\mathrm{min}$ ischemia and 120 -min reperfusion. Animals were excluded from the study when any of the following was observed: arterial blood pressure $<90 \mathrm{mmHg}$, pneumothorax, arrhythmia, or death before the scheduled time.

\section{Hemodynamics and cardiac function parameters}

Systolic blood pressure (SBP), diastolic blood pressure (DBP), HR, left ventricular systolic pressure (LVSP), left ventricular enddiastolic pressure (LVEDP), CVP, and LAP were recorded at the following time points: when the animal remained stable for $30 \mathrm{~min}$ after the threading operation (T0), before (T1) ischemia, after 30-min ischemia (T2), and after 120-min reperfusion (T3). LVDP was calculated as follows: LVDP = LVSP - LVEDP. Values of $+d p / d t m a x,-d p / d t m a x$, and t-dp/dtmax were calculated using the analyzing system of the RM6240 multichannel physiological signal detector for each time point.

Determination of endothelin 1 (ET-1), thromboxane A2 (TXA2), and platelet activating factor (PAF)

Two-milliliter samples of arterial blood were drawn from each rabbit at the T0, T1, T2, and T3 time points. The samples were centrifuged at $4000 \mathrm{r} / \mathrm{min}$ for $20 \mathrm{~min}$ to separate the plasma and kept at $-70^{\circ} \mathrm{C}$ for further study. Enzyme-linked immunosorbent assay was performed to determine the concentrations of TXA2, ET-1, and PAF in plasma. 
Distinguishing the area at risk (AAR), noreflow area, and MI area

After the experiment, $4 \%$ thioflavin $S$ (Beijing Zhongxi Pharmaceutical Co., Ltd.) was injected into the left atrium at a dose of $1 \mathrm{~mL} / \mathrm{kg}$. With 1-min intervals, the coronary artery was tied up again, followed by injection of $4 \mathrm{~mL}$ of $1 \%$ Evans blue (Hebei Bohai Biology Co., Ltd.) into the left auricle. After another 30 $\mathrm{s}, 5 \mathrm{~mL}$ of $10 \% \mathrm{KCl}$ was injected into the left auricle to stop the heartbeat, and the heart was taken out immediately and sliced along the short axis into five to eight pieces with equal thickness. The slices were photographed under normal light, in which the non-blue area represents the AAR and the blue area was the non-ischemic area. Under 365-nm ultraviolet light, blue fluorescence indicated the area with blood perfusion, whereas the area with no fluorescence was the no-reflow region. Finally, the samples were photographed again after immersion into 1.5\% 2,3,5-triphenyltetrazolium chloride solution and incubated in a $37-40^{\circ} \mathrm{C}$ water bath for $15 \mathrm{~min}$.

Statistical analysis

All statistical analyses were performed using SAS 8.0 statistical software (SPSS Inc., Chicago, IL, USA). Gaussian distributed measurement data were presented using $\bar{x}$ \pm s. One-way analysis of variance (ANOVA) for non-repeated measures was conducted, followed by Bonferroni's post hoc test, to test for differences in baseline hemodynamics. Data within groups were analyzed with oneway ANOVA for repeated measures, and data between groups were analyzed with twoway repeated measures ANOVA followed by Bonferroni's post hoc test. $P<0.05$ was considered to indicate statistical significance.

\section{- Results}

\section{Hemodynamic parameters}

At TO, the basal blood pressure, HR, LVSP, LVDP, LVEDP, +dp/dtmax, -dp/dtmax, and $\mathrm{t}$-dp/dtmax of the two groups were not significantly different $(P>0.05)$. However, at T3, the SBP, DBP, LVSP, LVDP, +dp/dtmax, and $-\mathrm{dp} / \mathrm{dtmax}$ of group $D$ were significantly higher than those of group IR $(P<0.05)$, whereas the values of $H R$, LVEDP, and $t$-dp/dtmax were lower in group $D$ than in group $\operatorname{IR~}(P<0.05$, Tables 1 and 2).

Table 1 - The results of SBP, DBP and HR $(n=8, \bar{x} \pm s)$.

\begin{tabular}{cccccc} 
Indicators & Group & T0 & T1 & T2 & T3 \\
\hline SBP & IR & $118 \pm 11$ & $102 \pm 8$ & $87 \pm 11^{\# \star}$ & $82 \pm 11^{\# \star \star}$ \\
& D & $120 \pm 13$ & $87 \pm 15^{\#}$ & $76 \pm 25^{\#}$ & $107 \pm 8^{\# \Delta^{\star \star}}$ \\
DBP & IR & $84 \pm 7$ & $54 \pm 4^{\# \star}$ & $51 \pm 8^{\# \star}$ & $48 \pm 9^{\# \star}$ \\
& D & $82 \pm 9$ & $53 \pm 8^{\# \star}$ & $44 \pm 13^{\# \star}$ & $60 \pm 11^{\# \Delta^{* \star}}$ \\
HR & IR & $254 \pm 21$ & $206 \pm 30$ & $196 \pm 19^{\# \star}$ & $198 \pm 23^{\# \Delta \star}$ \\
& D & $256 \pm 40$ & $170 \pm 16^{\#}$ & $175 \pm 32^{\# \star}$ & $175 \pm 19^{\#{ }^{\# \star}}$ \\
\hline
\end{tabular}

Note: Compared with Group IR, ${ }^{*} P<0.05$; compared with T0, ${ }^{\Delta} P<0.05$; compared withT1, ${ }^{\star} P<0.05$. 
Table 2 - The results of cardiac function $(n=8, \bar{x} \pm s)$.

\begin{tabular}{cccccc}
\hline Indicators & Group & TO & T1 & T2 & T3 \\
\hline LVSP & IR & $153.67 \pm 14.11$ & $131.74 \pm 7.65^{\Delta}$ & $112.65 \pm 1.13^{\Delta}$ & $107.70 \pm 14.69^{\star \star}$ \\
& D & $152.28 \pm 7.19$ & $115.12 \pm 6.59^{\# \Delta}$ & $110.40 \pm 16.19^{\Delta}$ & $130.77 \pm 12.70^{* \star}$ \\
LVEDP & IR & $126.90 \pm 2.73$ & $106.77 \pm 17.76$ & $99.78 \pm 21.49^{\Delta}$ & $98.37 \pm 16.13^{\Delta}$ \\
& D & $125.12 \pm 9.88$ & $84.74 \pm 10.75^{\# *}$ & $77.72 \pm 16.01^{*}$ & $84.08 \pm 6.97^{* \Delta}$ \\
LVDP & IR & $31.77 \pm 6.38$ & $32.09 \pm 4.65$ & $21.85 \pm 5.68^{\Delta \star}$ & $20.76 \pm 3.96^{\Delta \star}$ \\
& D & $32.15 \pm 2.67$ & $30.12 \pm 3.95$ & $25.67 \pm 0.18^{\Delta \star}$ & $28.39 \pm 3.42^{* \star \star}$ \\
+ dp/dtmax & IR & $1901.56 \pm 854.17$ & $1832.78 \pm 695.56$ & $1531.23 \pm 510.07^{\star}$ & $1448.32 \pm 350.84^{\star}$ \\
& D & $1915.91 \pm 151.22$ & $1897.67 \pm 217.41$ & $1743.33 \pm 316.34^{* \star}$ & $1791.95 \pm 20.67^{* \Delta}$ \\
-dp/dtmax & IR & $1106.38 \pm 102.58$ & $1082.02 \pm 34.55$ & $1052.70 \pm 55.86$ & $1004.99 \pm 37.72^{\star}$ \\
& D & $1151.05 \pm 60.91$ & $1099.06 \pm 53.32$ & $991.98 \pm 28.76^{* \Delta}$ & $1043.53 \pm 36.92^{* \star}$ \\
t-dp/dtmax & IR & $16.01 \pm 6.06$ & $21.93 \pm 4.55$ & $21.76 \pm 1.93$ & $37.56 \pm 8.40$ \\
& D & $14.41 \pm 2.15$ & $18.03 \pm 1.14$ & $21.57 \pm 1.62$ & $22.77 \pm 5.02$ \\
\hline
\end{tabular}

Note: Compared with Group IR, ${ }^{*} P<0.05$; compared with T0, ${ }^{\Delta} P<0.05$; compared withT1, ${ }^{\star} P<0.05$.

$A A R, M I$ area, and no-reflow area

The comparison of AAR between group IR and group $D$ showed no statistical significance ( $41 \pm 6 \%$ vs. $39 \pm 3 \%, P>0.05)$. The average $\mathrm{MI}$ area in group IR was significantly larger than that in group $D(48 \pm 5 \%$ vs. $18 \pm$ $3 \%, P=0.0317)$, whereas the average noreflow area was smaller ( $14 \pm 3 \%$ vs. $38 \pm 5 \%, P$ $=0.0213$ ).

Plasma concentration variations of ET-1, TXA2, and PAF

The ET-1, TXA2, and PAF levels of the two groups at T2 and T3 were significantly higher than those at the time point before ischemia $(P=0.0116)$, and when the values of the two groups were compared between T2 and T3, the differences were not statistically significant $(P>0.05)$. At the same time point, the ET-1 and TXA2 levels of the two groups was not significantly different $(P>0.05)$, whereas the PAF concentration in group $D$ was significantly higher than that in group IR $(P=$ 0.0317, Table 3).

Table 3 - The concentration of ET-1, TXA2 and NE (pg/ml, $\mathrm{n}=8, \bar{x} \pm \mathrm{s})$.

\begin{tabular}{cccccc}
\hline Indicators & Group & TO & T1 & T2 & T3 \\
\hline ET-1 & IR & $35.29 \pm 1.98$ & $36.20 \pm 0.98$ & $44.30 \pm 5.02^{\Delta \star}$ & $43.85 \pm 3.10^{\Delta \star}$ \\
& D & $36.29 \pm 1.10$ & $37.21 \pm 3.58$ & $45.28 \pm 2.31^{\Delta \star}$ & $41.64 \pm 4.04^{\Delta \star}$ \\
TXA2 & IR & $30.11 \pm 1.18$ & $30.31 \pm 0.76$ & $35.06 \pm 3.05^{\Delta \star}$ & $36.71 \pm 1.45^{\Delta \star}$ \\
& D & $30.15 \pm 0.38$ & $31.78 \pm 2.31$ & $36.70 \pm 1.24^{\Delta \star}$ & $36.85 \pm 2.32^{\Delta \star}$ \\
PAF & IR & $171.07 \pm 11.21$ & $181.97 \pm 33.27^{*}$ & $184.66 \pm 22.80^{* \Delta}$ & $178.86 \pm 12.48^{* \Delta}$ \\
& D & $173.07 \pm 26.04$ & $171.97 \pm 29.12$ & $246.97 \pm 39.07^{\Delta}$ & $239.95 \pm 20.57^{\Delta}$ \\
\hline
\end{tabular}

Note: Compared with Group IR, ${ }^{*} P<0.05$; compared with T0, ${ }^{\Delta} P<0.05$; compared withT1, ${ }^{\star} P<0.05$. 


\section{- Discussion}

The myocardial no-reflow area correlates with the $\mathrm{Ml}$ area. Specifically, an $80 \%$ necrotic myocardium was observed in the no-reflow area; therefore, it is important to reduce this extent to improve patients' prognosis ${ }^{15,16}$. This study demonstrated that dexmedetomidine administration could significantly reduce the no-reflow area in the myocardia with IRI and improve cardiac function. Dexmedetomidine can reduce myocardial oxygen demand, change the blood flow distribution in the epimyocardial and endomyocardial components of the ventricular wall, reduce HR and myocardial contractility, and alleviate myocardial ischemia. Although several studies have revealed that dexmedetomidine could slightly reduce cardiac blood flow, its influence on myocardial oxygen balance was not significant owing to the reduction in myocardial oxygen consumption ${ }^{17,18}$.

Our study demonstrated that after reperfusion, the $\mathrm{MI}$ and no-reflow areas of rabbits in group $D$ were significantly smaller than those in rabbits not treated with dexmedetomidine. Cardiac function was also found to be significantly enhanced when blood pressure, LVESP, and LVDP increased and LVEDP decreased. Although dexmedetomidine showed no remarkable impact on the systolic and diastolic functions of normal myocardia, it could improve the functions of myocardia with IRI, as shown by the lower $+d p / d t m a x$ and $-d p /$ dtmax in myocardia with IRI, which might be related to the reduction of $\mathrm{Ml}$ and no-reflow areas after dexmedetomidine administration. Our study revealed that dexmedetomidine premedication could protect myocardia with acute IRI, based on the factors described below.

$H R$ was reduced by dexmedetomidine before ischemia and after reperfusion

Myocardial oxygen supply was primarily determined by the oxygen content of arterial blood, coronary blood flow, and cardiac diastolic time. HR reduction can increase the diastole time and coronary blood flow. Even a slight increase in HR can markedly increase the myocardial oxygen consumption of patients with myocardial ischemic disease and break the balance, leading to myocardial ischemia or even MI. Therefore, HR reduction can alleviate myocardial ischemic injury and decrease the mortality risk of patients with acute $\mathrm{MI}^{19,20}$.

Many studies have demonstrated that the protective effect of dexmedetomidine on the myocardium results from HR reduction ${ }^{21,22}$. In our study, after dexmedetomidine administration, HR reduction occurred first, together with the decrease of systolic and diastolic pressure and myocardial work. Thioflavin $\mathrm{S}$ was used as a staining reagent for observing the no-reflow ischemic myocardial area, which was found to be significantly smaller in group $D$ than in group IR. Cardiac function was weaker in group $D$ than in group IR before ischemia (T1), appearing as corresponding changes of parameters such as blood pressure, HR, LVSP, LV, LVEDP, and $\pm d p / d$ tmax, which all resulted from the relief of cardiac load before ischemia caused by dexmedetomidine. Such cardiac function reduction might benefit cardiac function maintenance after ischemia reperfusion ${ }^{23}$, the detailed mechanism of which remains unclear.

After reperfusion, the HR in group $D$ was significantly lower than that in group IR, whereas LVDP was higher, indicating that dexmedetomidine administration could protect cardiac function, which might result from its HR-reduction effect. Moreover, DBP was remarkably higher in group $D$ than in group $I R$, which was one of the reasons why cardiac function in group D was higher. Increase in diastolic pressure can enhance the perfusion pressure in the ischemic myocardia, improve blood perfusion of the ischemic area, and reduce the myocardial no-reflow area. 


\section{Effect of dexmedetomidine on platelets during myocardial IRI}

Platelet activation is one of the main mechanisms of the no-reflow phenomenon in the myocardia with IRI. Ravn et al. ${ }^{24}$ found, in a pig myocardial IRI model, that platelet accumulation in AAR was twice as much as that in the non-hazardous area, and was even higher in the necrotic area, indicating the role of platelet accumulation in the myocardial necrosis process. A clinical study revealed that intermittent coronary artery occlusion occurred during the acute and thrombolytic stages of $\mathrm{MI}$, leading to repeated IRI. Coronary artery re-occlusion usually occurred in thrombogenic areas in the artery, which was activated by ruptured platelets ${ }^{25}$.

During the myocardial IRI process, platelets correlate strongly with vascular endothelial cells. TXA2 is an inflammatory mediator activated and released by platelets, and ET- 1 is a vasoconstrictive substance released by vascular endothelial cells. They interact with each other: ET-1 induces the release of TXA2; in turn, TXA2 can enhance the vasoconstrictive effect in the ischemic myocardia ${ }^{26}$. Application of ET-1 monoclonal antibody, ET-1 receptor blocker, and ET-1 transferase inhibitor can reduce the $\mathrm{Ml}$ area, improve cardiac function, increase myocardial blood flow, and finally protect the myocardium with IRI. Its possible mechanism may be related to the prevention of myocardial no-reflow, inhibition of ET-1related neutrophil granulocyte activation, and blocking of the interaction between ET-1 and the renin-angiotensin system ${ }^{27,28}$.

In this study, plasma TXA2, ET-1, and PAF levels were measured to evaluate the influence of dexmedetomidine on platelet activation during myocardial IRI, platelet accumulation, and vascular endothelial injury. The TXA2 and ET-1 levels increased in both groups after reperfusion; however, the values were not statistically different when compared between the two groups, suggesting that dexmedetomidine could not inhibit platelet activation and endothelin release. However, in this study, the PAF level in group $D$ was higher than that in group IR, indicating the activation effect of dexmedetomidine on platelets. Mustonen et al. ${ }^{28}$ also demonstrated that dexmedetomidine could promote platelet accumulation. Nevertheless, the protective effect of dexmedetomidine proved by our study suggests that the benefits of dexmedetomidine, such as its influence on the stress response and its role in protecting against oxidative damage, were more than its adverse effect due to platelet activation.

\section{Conclusion}

Dexmedetomidine can reduce the noreflow area of ischemic myocardia, improve cardiac function, and protect myocardia with ischemia-reperfusion injury.

\section{References}

1. Braunwald E, Kloner RA. Myocardial reperfusion: a double-edged sword? J Clin Invest. 1985;76(5):1713-9. PMID: 4056048.

2. Boyle EM Jr, Pohlman TH, Cornejo CJ, Verrier ED. Endothelial cell injury in cardiovascular surgery: ischemia-reperfusion. Ann Thorac Surg. 1996;62(6):1868-75. PMID: 8957415.

3. Park JL, Lucchesi BR. Mechanisms of myocardial reperfusion injury. Ann Thorac Surg. 1999;68(5):1905-12. PMID: 10585102.

4. Williams FM. Neutrophils and myocardial reperfusion injury. Pharmacol Ther. 1996;72(1):1-12. PMID: 8981567.

5. Reffelmann T, Kloner RA. Microvascular alterations after temporary coronary artery occlusion: the no-reflow phenomenon. J Cardiovasc Pharmacol Ther. 2004;9(3):16372. PMID: 15378136

6. Michaels AD, Gibson CM, Barron HV. Microvascular dysfunction in acute myocardial infarction: focus on the roles of platelet and inflammatory mediators in 
the no-reflow phenomenon. Am J Cardiol. 2000;85(5A):50B-60B. PMID: 11076131.

7. Kloner RA, Ganote CE, Jennings RB. The "no-reflow"phenomenon after temporary coronary occlusion in the dog. J Clin Invest. 1974;54(6):1496-508. PMID: 4140198.

8. Ren F, Mu N, Zhang X, Tan J, Li L, Zhang C, Dong $M$. Increased platelet-leukocyte aggregates are associated with myocardial no-reflow in patients with ST elevation myocardial infarction. Am J Med Sci. 2016;352(3):2616. PMID: 27650230.

9. Kloner RA. No-reflow phenomenon: maintaining vascular integrity. J Cardiovasc Pharmacol Ther. 2011;16(3-4):244-50. PMID: 21821523.

10.Niccoli G, Cosentino N, Spaziani C, Minelli S, Fracassi F, Crea F. New strategies for the management of no-reflow after primary percutaneous coronary intervention. Expert Rev Cardiovasc Ther. 2011;9(5):615-30. PMID: 21615325.

11.Tritto I, Zuchi C, VitaleS, Ambrosio G. Therapy against reperfusion-induced microvascular injury. Curr Pharm Des. 2013;19(25):458696. PMID: 23270550.

12.Okada H, Kurita T, Mochizuki T, Morita $\mathrm{K}$, Sato S. The cardioprotective effect of dexmedetomidine on global ischaemia in isolated rat hearts. Resuscitation. 2007;74(3):538-45. PMID: 17391832.

13.Ammar AS, Mahmoud KM, Kasemy ZA, Helwa MA. Cardiac and renal protective effects of dexmedetomidine in cardiac surgeries: A randomized controlled trial. Saudi J Anaesth. 2016;10(4):395-401. PMID: 27833481.

14.Kamibayashi T, Maze M. Clinical uses of alphal2-adrenergicagonists. Anesthesiology. 2000;93(5):1345-9. PMID: 11046225.

15. Reffelmann T, Hale SL, Li G, Kloner RA. Relationship between no reflow and infarct size as influenced by the duration of ischemia and reperfusion. Am J Physiol Heart Circ Physiol. 2002;282(2):H766-72. PMID: 11788428.

16.Wallace AW, Galindez D, Salahieh A, Layug EL, Lazo EA, Haratonik KA, Boisvert DM, Kardatzke D. Effect of clonidine on cardiovascular morbidity and mortality after noncardiac surgery. Anesthesiology. 2004;101(2):284-93. PMID: 15277909.

17. Roekaerts PM, Prinzen FW, de Lange
S. Coronary vascular effects of dexmedetomidine during reactive hyperemia in the anesthetized dog. J Cardiothorac Vasc Anesth. 1996;10(5):61926. PMID: 8841870.

18. Lawrence CJ, Prinzen FW, de Lange S. The effect of dexmedetomidine on the balance of myocardial energy requirement and oxygen supply and demand. Anesth Analg. 1996;82(3):544-50. PMID: 8623959.

19.Perne A, Schmidt FP, Hochadel M, Giannitsis E, Darius H, Maier LS, Schmitt C, Heusch $G$, Voigtländer T, Mudra H, Gori T, Senges J, Münzel T, German Chest Pain Unit Registry. Admission heart rate in relation to presentation and prognosis in patients with acute myocardial infarction. Herz. 2016;41(3):233-40. PMID: 26411426.

20.Ceconi C, Cargnoni A, Francolini G, Parinello $G$, Ferrari R. Heart rate reduction with ivabradine improves energy metabolism and mechanical function of isolated ischaemic rabbit heart. Cardiovasc Res. 2009;84(1):7282. PMID: 19477966.

21.Willigers HM, Prinzen FW, Roekaerts PM, de Lange S, Durieux ME. Dexmedetomidine decreases perioperative myocardial lactate release in dogs. Anesth Analg. 2003;96(3):657-64. PMID: 12598239.

22.Penttilä J, Helminen A, Anttila $M$, Hinkka S, Scheinin $H$. Cardiovascular and parasympathetic effects of dexmedetomidine in healthy subjects. Can J Physiol Pharmacol. 2004;82(5):359-62. PMID: 15213737.

23. Kim YJ, Lim HJ, Choi SU. Effect of propofol on cardiac function and gene expression after ischemic-reperfusion in isolated rat heart. Korean J Anesthesiol. 2010;58(2):153-61. PMID: 20498794.

24.Ravn HB, Møldrup U, Ilkjaer LB, Chew $M$, Jensen $L$, Johnsen $S$, Birk-Sørensen $L$, Tønnesen E, Hjortdal VE. A new model for evaluation of thrombosis and ischaemia/ reperfusion injury. APMIS. 2000;108(5):3739. PMID: 10937776.

25.Zaugg CE, Hornstein PS, Zhu P, Simper D, Lüscher TF, Allegrini PR, Buser PT. Endothelin1 -induced release of thromboxane $A 2$ increases the vasoconstrictor effect of endothelin-1 in postischemic reperfused rat hearts. Circulation. 1996;94(4):742-7. PMID: 8772697. 
26.Pernow J, Wang QD. Endothelin in myocardial ischaemia and reperfusion. Cardiovasc Res. 1997;33(3):518-26. PMID: 9093521.

27.Saito T, Fushimi E, Abe T, Kimura Y, Takahashi K, Kudo Y, Nakagomi A, Miura M. Augmented contractile response to endothelin and blunted endothelium-dependent relaxation in post-ischemic reperfused coronary arteries. Jpn Circ J. 1992;56(7):657-70. PMID: 1495159.

28. Mustonen P, Savola J, Lassila R. Atipamezole, an imidazoline-type alpha(2)adrenoceptor inhibitor, binds to human platelets and inhibits their adrenalineinduced aggregation more effectively than yohimbine. Thromb Res. 2000;99(3):231-7. PMID: 10942789.

\section{Correspondence:}

Jianjun Ren,

Department of Anesthesia, The Second Hospi-

tal of Hebei Medical University

Shijiazhuang 050000 Hebei China

Phone: +8618630138191

cnjianjunren@163.com

Received: Sep 16, 2017

Review: Nov 17, 2017

Accepted: Dec 20, 2017
Conflict of interest: none

Financial source: none
${ }^{1}$ Research performed at Department of Central Laboratory, the Second Hospital of Hebei Medical University, Shijiazhuang, China. 